\title{
Extreme Polysemie - der Fall ziehen
}

\section{Probleme und Methoden}

Hundsnurscher (1996) hat mit einer umfangreichen Liste von Beispielen für Verwendungsmöglichkeiten des Verbs ziehen auf das bemerkenswerte Bedeutungsspektrum dieses Verbs aufmerksam gemacht und auch schon wichtige Hinweise auf Zusammenhänge zwischen diesen Verwendungsweisen gegeben.' Der vorliegende Beitrag ist ein Gegenstuck zu meiner Untersuchung des Verwendungsspektrums von scharf in Fritz (1995). ${ }^{2}$ Dort bin ich näher auf den bedeutungstheoretischen Status des Begriffs der Verwendungsweise und auf Probleme und Methoden der Unterscheidung von Verwendungsweisen eingegangen, so dass ich im vorliegenden Beitrag die dort explizierten theoretischen und methodischen Annahmen nur andeuten will (vgl. auch Fritz 1998, $11 \mathrm{ff}$.). ${ }^{3}$

Um einen ersten Eindruck von der Vielfalt der Verwendungsweisen von ziehen zu geben, fuhre ich zunächst einige Verwendungsbeispiele an, bei denen man annehmen könnte, dass sie jeweils eine eigene Verwendungsweise repräsentieren.

(1) Das Kind zieht am Turgriff - aber die Tur bewegt sich nicht

(2) Das Kind zieht den Wagen

(3) Der Ritter zieht das Schwert aus der Scheide

(4) Die Plastikmasse lässt sich ziehen

(5) Der Magnet zieht nicht mehr

(6) Der Motor der R1100 zieht wie der Teufel

(7) Der Nachbar zieht eine Mauer

(8) Die Mauer zieht sich entlang der Grundstucksgrenze

(9) Schroder zieht an seiner Havanna

(10) Es zieht

(11) Die Wolken ziehen am Himmel

(12) Die Parlamentarier ziehen nach Berlin

1 Hundsnurscher, Franz: Wortsemantik aus der Sicht einer Satzsemantik, in: Hundsnurscher, Franz; Weigand, Edda (Hgg.): Lexical Structures and Language Use. Bd. 1. Tubingen 1996, S. 39-51. (Beitrăge zur Dialogforschung 8)

Fritz, Gerd: Metonymische Muster und Metaphernfamilien. Bemerkungen zur Struktur und Geschichte der Verwendungsweisen von scharf, in: Hindelang, Gotz; Rolf, Eckard; Zillig, Werner (Hgg.): Der Gebrauch der Sprache. Festschrift fur Franz Hundsnurscher zum 60. Geburtstag. Munster 1995, S. 77-107.

3 Fritz, Gerd: Historische Semantik. Stuttgart/Weimar 1998. (Sammlung Metzler 313) 
Schon diese kurze Liste lässt erkennen, dass ziehen ein erstaunliches Verwendungspotential hat. Aus der Sicht des beschreibenden Semantikers gibt es nun, grob gesprochen, drei Auffassungen, wie man mit einem derartigen Befund umgehen kann:

(i) Man nimmt an, dass jede dieser Verwendungsweisen isoliert zu beschreiben ist.

(ii) Man nimmt an, dass diese Verwendungsweisen zwar alle einzeln etabliert sind, dass es aber zwischen ihnen systematische Zusammenhănge gibt, die es zu zeigen gilt.

(iii) Man nimmt an, dass es eine Grundbedeutung von ziehen gibt, von der alle Einzelverwendungen konversationell abzuleiten sind (die minimalistische Strategie).

Strategie (i) ist methodisch resignativ, weil sie sich der Möglichkeit verschließt, eine zumindest partielle Einheit der Bedeutung von ziehen zu rekonstruieren. Sie ist auch empirisch unbefriedigend, weil sie voraussetzt, dass die Sprecher keine Zusammenhänge zwischen diesen Verwendungsweisen sehen und folglich auch jede Verwendungsweise einzeln lernen müssen. Das wäre ein sehr unökonomisches System, das auch unserer Intuition von der (zumindest partiellen) Einheit der Bedeutung eines Wortes wie ziehen widerspricht.

Strategie (iii) ist methodisch in vielen Fällen akzeptabel, führt allerdings zu dem Problem, dass man oft weite Ableitungswege gehen muss, um von einer angenommenen Grundbedeutung zur Deutung einer bestimmten Verwendung zu gelangen. Empirisch ist sie ebenfalls unbefriedigend, weil sie außer Acht lässt, dass viele Verwendungsweisen fest etabliert sind und dass deshalb entsprechende Verwendungen (Sprecherperspektive) und Deutungen (Hörerperspektive) routinisiert sind und nicht ad hoc konversationell gefunden werden müssen.

Strategie (ii) vermeidet die genannten Probleme von (i) und (iii). Das Problem der weiten Deutungswege umgeht diese Strategie durch die Annahme, dass nicht alle Verwendungen direkt von einer Grundbedeutung abgeleitet werden müssen, sondern dass es etablierte Zwischenglieder zwischen bestimmten prototypischen Verwendungsweisen und verschiedenen Einzelverwendungen gibt, so dass diese Zwischenglieder den Sprechern als Deutungsgrundlage für bestimmte Einzelverwendungen dienen können. Empirisch setzt diese Strategie voraus, dass die Sprecher Zusammenhänge zwischen diesen oder wenigstens einigen dieser Verwendungsweisen sehen und es in diesem Sinne eine gewisse Einheit der Bedeutung gibt. Bei dieser Betrachtungsweise wäre zieken ein sehr ökonomisches Werkzeug, ein Multifunktionsinstrument. Mit der Wahl dieser Strategie, der ich in diesem Beitrag folgen will, handelt man sich natürlich die Aufgabe ein, mögliche Zusammenhänge zu zeigen und plausibel zu machen. Zur (partiellen) Lösung dieser Aufgabe möchte ich im Folgenden einen Versuch machen.

Von einer partiellen Lösung spreche ich in zweierlei Hinsicht. Erstens kann ich auf dem hier verfügbaren Raum nur einen Teil der vielfältigen Beziehungen behandeln, die man sehen kann. Und zweitens vermute ich bei einer ganzen Anzahl von Verwendungsweisen, dass der Zusammenhang mit anderen Verwendungsweisen abgerissen ist. Dies gilt insbesondere fur einige feste Verbindungen, u. a. die Verwendung als Funktionsverb: 
(13) Er hat Bilanz gezogen

(14) Er zieht die Konsequenzen

(15) Auch diese Möglichkeit mussen wir in Betracht/in Erwägung ziehen

Derartige Verwendungsweisen lernen die Sprecher normalerweise relativ spăt, und zwar als isolierte, quasi-idiomatische Verbindungen. Auf diese Frage werde ich in Abschnitt 5 nochmals kurz eingehen.

\section{Beschreibungsgrundlagen}

Mit dem Verb ziehen und seinen Aktanten kann man eine Reihe von Ereignistypen (Szenen) charakterisieren, von denen ich annehme, dass sie eine Familie bilden, also in irgendeinem Sinne systematisch zusammenhängen. Die folgende Beschreibung ist der Versuch einer rationalen Rekonstruktion von Teilen eines solchen Familienzusammenhangs, d. h. eine Beschreibung, die plausibel machen soll, wie Sprecher derartige Zusammenhänge sehen könnten. Die stärkere Behauptung, dass die Sprecher die Zusammenhănge tatsächlich so sehen, ist naturlich eine empirische Behauptung, die also auch empirisch zu belegen wäre. Diesen Nachweis kann ich hier nicht führen. Allerdings deuten informelle Befragungen darauf hin, dass manche Sprecher manche dieser Zusammenhänge tatsächlich so sehen, allerdings auch darauf, dass verschiedene Sprecher die $\mathrm{Zu}$ sammenhänge $z$. $T$. verschieden sehen.

Das Ziel meiner Beschreibung besteht primär darin, Wissensbestände und semantische Verfahren zu eruieren, die die Sprecher bei der Deutung von Verwendungen von ziehen nutzen können. Wie schon erwähnt, setze ich voraus, dass es furr viele gebräuchliche Verwendungsweisen dieses Verbs routinisierte Deutungen gibt. Aber auch für diese Fälle erscheint die Annahme plausibel, dass die ad-hoc-Deutung entsprechender Verwendungen auf der Folie bestimmter zentraler Verwendungsweisen möglich wäre. Die wichtigsten Wissensbestănde und semantischen Verfahren will ich an dieser Stelle schon erwăhnen, um die Aufmerksamkeit auf diese Aspekte der folgenden Beschreibung zu lenken:

(i) stereotypes Wissen aber eine Anzahl von grundlegenden Ereignistypen, die mit ziehen charakterisiert werden können, und deren Hauptaspekte,

(ii) stereotypes Wissen aber bestimmte Arten von Gegenstănden (bewegliche Gegenstănde, elastische Gegenstănde etc.),

(iii) die Mőglichkeit, Aspekte der Ereignistypen mit bestimmten Aktanten des Verbs explizit auszudrucken oder, z. B. durch elliptischen Gebrauch, implizit zu lassen,

(iv) die Moglichkeit, durch Fokussierung von Aspekten, durch Hinzufugen von Aspekten und durch Ausblenden von Aspekten verwandte Ereignistypen zu charakterisieren,

(v) die Fahigkeit, metaphorische Verfahren anzuwenden. 


\section{Erster Beschreibungsschritt: Zwei Prototypen}

In einem erster Schritt meiner Beschreibung des Gebrauchs von ziehen gehe ich davon aus, dass die Sprecher ein stereotypes Wissen uber zwei grundlegende Ereignistypen (E1) und (E2) haben, die ihrerseits verwandt sind, von denen einzelne Aspekte fokussiert oder ausgeblendet sein können und an die zusătzliche Aspekte angelagert werden können. ${ }^{4}$

(E1) A ubt auf einen Gegenstand (gegen einen Widerstand) eine Kraft in Richtung auf A hin aus (mit der Intention, den Gegenstand zu A hinzubewegen).

(E2) A ubt auf einen Gegenstand (gegen einen Widerstand) eine Kraft in Richtung auf A hin aus, so dass sich der Gegenstand in der Bewegungsrichtung von A mitbewegt.

Die Möglichkeit zur Charakterisierung wichtiger Aspekte dieser beiden Ereignistypen ergibt zwei prototypische Verwendungsweisen, die durch die Beispiele (16) und (17) reprăsentiert sind. Als prototypische Besetzung der Subjektsposition "A" kann ein Agens-Ausdruck wie in (16) gelten:

(16) Das Kind zieht am Turgriff

(17) Das Kind zieht den Wagen

Den Kontrast der beiden Verwendungsweisen kann man hervorheben, wenn man die Beispielsătze einander angleicht, also etwa (18) und (17) wăhlt:

(18) Das Kind zieht am Wagen

Syntaktisch auffallend ist der Unterschied von Akkusativ-Ergănzung in (17) und präpositionaler Ergänzung in (16) bzw. (18). Dabei gehört in Făllen wie (16) oder (18) der Angriffspunkt der Kraft, der mit einer Präpositionalphrase ausgedrückt wird (am Türgriff, am Wagen, an den Haaren etc.) im Sinne von Heringer $(1984,37)$ zweifellos zu den Grundaspekten der ziehen-Szene. ${ }^{5}$ Dieser Aspekt kann auch bei Äußerungen nach dem Muster (E2) ausgedrückt werden, wie (19) zeigt:

(19) Das Kind zieht die Puppe an den Haaren hinter sich her

Den Zusammenhang von (E1) und (E2) kann man auf unterschiedliche Art beschreiben: Geht man von (E1) aus, dann sind der Bewegungs- und der Richtungsaspekt in (E2) zusätzliche Aspekte, die aufgrund unseres Wissens uber die Wirkungen der Kraftanwendung auf bewegliche Gegenstände bei der Deutung einer entsprechenden Äußerung eingefuhrt werden. Geht man dagegen von (E2) aus, so werden bei einer Deutung nach (E1) diese Aspekte ausgeblendet.

4 Die Formulierungen, die ich verwende, um die Ereignistypen und die Zusammenhănge ihrer Aspekte explizit zu machen, klingen z. T. etwas kunstlich. Darin scheint sich bei semantischen Beschreibungen von Basislexemen eine gewisse Notlage auszudrucken, die mit der Frage der Dosierung des Grades der Explizitheit zusammenhangt. Die Funktion der Formulierungen mag jedoch ihre Form rechtfertigen.

5 Heringer, Hans Jurgen: Neues von der Verbszene, in: Stickel, Gerhard (Hg.): Pragmatik in der Grammatik. Jahrbuch 1983 des Instituts furr deutsche Sprache. Dusseldorf 1984, S. 3464. 
Unabhängig von der Konstruktion dieses Zusammenhangs benutze ich beide Typen als Prototypen, von denen aus andere Verwendungen gedeutet werden können. Eine auffallende Beobachtung ist, dass in manchen Fällen mit der Ausblendung des Bewegungsaspekts ein Misserfolg signalisiert werden kann, wie der Vergleich von (20) und (21) bzw. (22) und (23) zeigt. Dies spricht für die Berücksichtigung des Aspekts „mit der Intention, den Gegenstand zu A hinzubewegen" in der Beschreibung (E1).

(20) A zieht den Wagen

(21) A zieht am Wagen

(22) A zieht die Notbremse

(23) A zieht an der Notbremse

4. Weitere Beschreibungsschritte: Die nähere Verwandtschaft

Im folgenden Beschreibungsteil versuche ich, Zusammenhänge einiger etablierter Verwendungsweisen von ziehen mit den beiden genannten prototypischen Verwendungsweisen zu zeigen. Von den so eingefuhrten Verwendungsweisen ausgehend zeige ich anhand von Beispielen weitere Verzweigungen im Familienbaum von ziehen. ${ }^{6}$

\subsection{Das Extraktions-Muster}

Von (E1) ausgehend kann man mit einer Präpositionalphrase mit aus einen zusătzlichen Aspekt einfuhren, so dass man einen Spezialfall des Bewegungstyps (E2) erhält. Man kann mit der Verwendung von ziehen und geeigneten Aktanten ausdrucken, dass eine Person eine Kraft auf einen Gegenstand ausubt, der in sich in einem Behältnis befindet, so dass der Gegenstand aus dem Behältnis herausbewegt wird.

(24) Er zieht das Schwert aus der Scheide

(25) Er zieht den Korken aus der Flasche

Vor allem in Bezug auf Schusswaffen finden wir zwei Stufen des elliptischen Gebrauchs (27)/(28):

(26) Er zog den Colt aus dem Halfter

(27) Er zog den Colt

(28) Er zog (schneller als Billy the Kid)

6 Fur den vorliegenden Beitrag konnte ich auf Beispielmaterial zuruckgreifen, das K. Lynker in ihrer Examensarbeit zu ziehen aus dem elektronischen Corpus der SPIEGEL-Ausgaben von 1996 gewonnen hat. Nutzliches Datenmaterial liefert auch DIE ZEIT auf CD-ROM (1995-1998). 
Das Gegenstïck zu (27) ist der Normalfall bei (29), wo mitverstanden wird, dass der Zahn aus seiner Befestigung im Kiefer herausbewegt wird. ${ }^{7}$

(29) Der Zahnarzt zieht den Backenzahn

Auch in der Verwendung (30) ist der Bezug auf einen Behälter, den Automaten, mitverstanden:

(30) Er zieht sich eine Schachtel Zigaretten

Als eine Übertragung vom Personen-Prototyp in (24)-(30) kann man (31) deuten:

(31) Die Pflanzen ziehen Năhrstoffe aus dem Boden

Allerdings könnte man hier auch einen Zusammenhang mit dem Inhalationstyp A zieht den Rauch in die Lunge (61) sehen. Von hier fuhrt möglicherweise ein weiterer metaphorischer Pfad zu (32):

(32) Er zieht Nutzen aus seiner Mitgliedschaft

Statt in einem Behältnis kann der Gegenstand auch in einer relativ festen oder klebrigen Masse stecken, aus der der Gegenstand dann herausbewegt wird:

(33) Munchhausen zog sich an seinem eigenen Zopf aus dem Sumpf

Auch dieses Muster kann man metaphorisch nutzen. Wenn man annimmt, dass es eine unangenehme oder auch gefährliche Situation ist, in einer derartigen Masse zu stecken, kommt man zu einer möglichen Deutung von (34):

(34) Die Manager ziehen sich aus der Verantwortung

Alternativ könnte man für (34) auch eine metaphorische Verwendung von (35) als Deutungshintergrund nehmen:

(35) Er zieht den Kopf aus der Schlinge

Die Tatsache, dass sich mehrfach verschiedene Deutungswege für das Verständnis einer bestimmten Verwendung anbieten, zeigt, dass eine Verwendungsweise durch unterschiedliche Zusammenhänge mit anderen Verwendungsweisen gestützt sein kann. Bei einer sehr feinkörnigen Beschreibung kăme man dabei möglicherweise auch zu der Einsicht, dass je nach bevorzugtem Deutungshintergrund bei unterschiedlichen Sprechern feine Gebrauchsunterschiede $\mathrm{zu}$ erkennen sind, die jedoch in der alltäglichen Praxis und bei einer grobkörnigeren Beschreibung nicht $\mathrm{zu}$ Buche schlagen. Insgesamt scheint eine derartige mehrfache Deutbarkeit von Verwendungen zur Robustheit des Systems der Verwendungsweisen beizutragen. Diese Betrachtungsweise gibt auch einen Hinweis darauf, warum sich Kinder ein derartig komplexes System, abgesehen vom

7 Das Zahnarztbeispiel zeigt wieder ein Problem der Beschreibungssprache. Wir würden den Mund oder auch den Raum im Kiefer, in dem der Zahn sitzt, naturlich nicht als Behältnis oder Behalter bezeichnen. Andererseits scheinen Behaltnisse aber doch den Prototyp fur das Extraktions-Modell abzugeben, das dann flexibel angewendet werden kann. Alternativ könnten wir das Zahnarztbeispiel aber auch nach dem verwandten Muster (33) deuten, wobei dann der Aspekt der Befestigung des Zahns fokussiert wăre. 
fachsprachlichen Teil, relativ schnell aneignen. Da es unterschiedliche Deutungswege gibt, kann sich das Kind opportunistisch den nächstliegenden wăhlen und damit in vielen Fällen zu einem befriedigenden Deutungsresultat kommen.

Ein methodisch lehrreiches Beispiel ist auch die Verwendung (36):

(36) Der Tee muss noch ziehen ,die Teeblatter mussen noch im heißen Wasser bleiben

Man könnte versuchen, (36) auf der Folie von Verwendungen wie (16) zu deuten als eine spezielle Anwendung des Extraktions-Musters. Das mag als historische Rekonstruktion zutreffend sein, aber in der Praxis der Sprecher scheint dieser Zusammenhang keine Rolle zu spielen. Damit muß der Versuch, einen Zusammenhang mit anderen Verwendungsweisen zu zeigen, hier wohl aufgegeben werden. Also dürfte (36) ein Beispiel für eine isolierte Verwendungsweise sein, die als solche etabliert ist.

\subsection{Das Verformungs-Muster}

Ein zusätzlicher Aspekt wird auch eingeführt bei der Anwendung von ziehen in Bezug auf elastische Gegenstände. Aufgrund des Alltagswissens, dass sich derartige Gegenstände bei Kraftanwendung ausdehnen oder anderweitig verformen können, wird eine Deutung nach folgendem Muster möglich: Eine Person übt (gegen einen Widerstand) eine Kraft auf einen elastischen Gegenstand aus, so dass sich der Gegenstand in einer bestimmten Richtung verformt.

(37) A zieht den Kaugummi

(38) A zieht den Draht

Diese Verwendungsweise findet sich häufig mit implizitem Agens in der Konstruktion mit lässt sich:

(39) Die Plastikmasse lässt sich ziehen ,man kann die Plastikmasse verformen“

Wenn reine Verformung ohne Agens ausgedrückt werden soll, wird die reflexive Konstruktion verwendet:

(40) Das Holz zieht sich

Auch vom Ausdehnungs-Muster ist eine metaphorische Übertragung gebräuchlich, von der räumlichen auf die zeitliche Ausdehnung:

(41) Die Sitzung zieht sich (in die Lange)

Möglicherweise kann man mit dem Ausdehnungs-Muster auch die Verwendung (42) in Verbindung bringen:

(42) Er zieht Tomaten aus Setzlingen 


\subsection{Anziehungskraft: das Magnet-Modell}

Ausgehend von (E1) kann die Anziehungskraft eines Gegenstands auf andere Gegenstănde fokussiert werden. Prototyp eines derartigen anziehenden Gegenstands ist der Magnet: ${ }^{8}$

(43) Der Magnet zieht nicht mehr

Das Magnet-Modell kann in verschiedener Weise metaphorisch genutzt werden, um die Attraktivität eines Gegenstands zu charakterisieren. Auch hier sind die syntaktischen Varianten bemerkenswert:

(44) Der alte Film zieht immer noch

(45) Nutzwert zieht beim Bundesburger

(46) Viele Leute zieht es in die Sudsee

(47) Der Carisma von Mitsubishi zieht keine bewundernden Blicke auf sich

\subsection{Kraftentfaltung}

Von Fahrzeugmotoren kann mit ziehen die reine Kraftentfaltung prädiziert werden:

(48) Der Motor der R1100 zieht wie der Teufel

Diese Verwendungsweise, mit der ein Kraftpotential angegeben wird, könnte man von (E1) ableiten. Allerdings ist auch eine Verknüpfung mit dem Bewegungs-Prototyp (E2) denkbar - aufgrund des Wissens, dass diese Kraftentfaltung normalerweise eine Beschleunigung des Fahrzeugs bewirkt, wenn die Kupplung losgelassen wird. Bei dieser Deutung wăre der Aspekt der Bewegung des Fahrzeugs mitverstanden.

\subsection{Herstellung eines Gegenstands mit einer bestimmten Verlaufslinie}

Auch folgende Variante eines Ereignistyps kann mit ziehen charakterisiert werden: Jemand erstellt einen Gegenstand. Beim Arbeitsvorgang bewegt sich der Herstellende (mit seinem Werkzeug) in einer bestimmten Richtung, so dass der entstehende Gegenstand in dieser Richtung eine Linie bildet.

(49) Der Nachbar zieht eine Mauer/einen Zaun/einen Graben

Bei dieser Verwendung von ziehen ist der Zusammenhang mit (E1) schon etwas weniger eng. Der Aspekt der Kraftentfaltung scheint mehr oder weniger ausgeblendet zu sein. Vielleicht kann man sagen, dass er in den Aspekt des Arbeits-

8 An dieser Stelle kőnnte man ein komplexes Thema anschließen, das in diesem Beitrag ganz ausgeklammert wird, namlich die Frage des semantischen Zusammenhangs zwischen dem Simplexverb ziehen und den Partikelverben wie anziehen, aufziehen, herausziehen etc. In manchen Fallen bietet sich die Hypothese an, dass Simplexverwendungen elliptische Versionen von Partikelverb-Verwendungen sind. 
vorgangs integriert ist. Im Vordergrund steht das Produzieren eines Gegenstands, dessen Hauptdimension die Lănge ist. Eine nahe verwandte Verwendungsweise repräsentiert (50):

(50) Adrian zieht einen Strich

\subsection{Verlaufslinie eines Gegenstands}

Im Anschluss an 4.5 kann mit der Verwendung von ziehen auch die Verlaufslinie eines Gegenstands fokussiert werden, ohne dass ein Herstellungsvorgang erwăhnt oder uberhaupt angenommen wird. Dazu dient der reflexive Gebrauch.

(51) Die Mauer zieht sich entlang der Grundstücksgrenze

(52) Der Fluss zieht sich durch die Ebene

\subsection{Bewegung in eine bestimmte Richtung}

Gehen wir nun von (E2) aus, so läßt sich bei der Verwendung von ziehen der Aspekt der Bewegung in eine bestimmte Richtung fokussieren und der Aspekt der externen Kraftanwendung ausblenden. Auf diese Weise kann man folgende Szene charakterisieren: Personen, Tiere oder andere Gegenstănde bewegen sich in eine bestimmte Richtung.

(53) Die Wolken ziehen (am Himmel)

Grammatisch zeigt sich die Ausblendung des Aspekts der externen Kraftanwendung in einer Agensverschiebung. Wahrend in den prototypischen Verwendungen der Art Das Kind zieht am Türgriff und Das Kind zieht den Wagen mit dem Subjektsausdruck jeweils auf eine Person (oder einen anderen Gegenstand) Bezug genommen wird, die die Bewegung durch externe Wirkung herbeiführt, wird in (53) bis (57) mit dem Subjektsausdruck jeweils auf die Gegenstände Bezug genommen, von denen die Bewegung selbst prädiziert wird.

(54) Abends zieht er mit seinen Mitarbeitern durch die Berliner Kneipen

$\mathrm{Zu}$ unserem Alltagswissen über Bewegungen gehort, dass sie von einem Ausgangsort zu einem Zielort verlaufen. Bei der hier beschriebenen Verwendung von ziehen gehört die Angabe des Zielorts durch eine Prăpositionalphrase mit nach oder in zur Standardsituation:

(55) Das Gewitter zieht nach Westen

(56) Die Schwalben ziehen nach Suden

(57) Der Zirkus zieht in die năchste Stadt

Zusătzlich kann der Ausgangsort der Bewegung durch eine Prăpositionalphrase mit von ausgedruckt werden:

(58) Der Zirkus zieht von Gießen nach Wetzlar 
Einen Sonderfall des Ortswechseltyps (57) haben wir in Satz (59), mit dem wir ausdricken, dass Leute den Wohnort wechseln, m. a. W. sich mit ihrer Wohnungseinrichtung an einen Ort begeben, an dem sie längere Zeit zu bleiben beabsichtigen.

(59) Die Parlamentarier ziehen nach Berlin

\subsection{Bewegung von flüchtigen Materien}

Neben den beweglichen und den elastischen Gegenständen bilden flüchtige Materien wie Luft oder Rauch (ähnlich: flüssige Materien) eine weitere interessante Gruppe von Gegenständen, die durch Kraftanwendung in Bewegung versetzt werden können. Diese Beschreibungskategorie liegt quer zu der Unterscheidung von (E1) und (E2). Von beiden Prototypen lassen sich Sonderverwendungen beim Bezug auf flüchtige Gegenstände ableiten, z. B. (60) und (61). Dabei lässt sich die Art der Kraftanwendung und der Bewegung jeweils aufgrund des Wissens über den Umgang mit derartigen flüchtigen Materien deuten:

(60) Schroder zieht an seiner Havanna

Hier wird mitverstanden, dass es Rauch ist, der inhaliert wird, wenn auch dieser Aspekt der Szene nicht ausgedrückt ist:

(61) A zieht den Rauch in die Lunge

Als eine Art metaphorische Verwendung lässt sich (62) von den eben erwăhnten Verwendungsweisen ableiten:

(62) Der Kamin zieht wieder besser

Man kann damit ausdrücken, dass der Kamin aufgrund seiner Eigenschaften die Bewegung des Rauchs, d. h. seinen Abzug, bewirkt bzw. ermöglicht.

In einen Zusammenhang mit Verwendungen vom Typ (62) gehört vielleicht auch die Verwendungsweise (63), die aufgrund der Valenzeigenschaften des Ausdrucks der Familie von es friert, es regnet, es schneit zugeordnet werden kann:

(63) Es zieht

Ähnlich wie bei den anderen nullwertigen Verben auch wird hier die Luftbewegung als eine Art Umweltereignis ohne Agens thematisiert. Eine Verwandtschaft könnte man hier auch zum reinen Bewegungsmuster sehen (Die Warmluft zieht nach Osten, Der Rauch zieht durchs Zimmer). 
5. Metaphorische, idiomatische und isolierte Verwendungsweisen

In der bisherigen Beschreibung wurden schon an verschiedenen Stellen metaphorische Verwendungsweisen erwähnt. Grundsätzlich kann man natürlich alle Verwendungsweisen zur Basis einer metaphorischen ad-hoc-Verwendung machen. Beispielsweise könnte man (64) metaphorisch verwenden, um zu verstehen zu geben, dass ein Politiker seine Fraktionskollegen kräftig ermahnt hat:

(64) Er hat seine Fraktionskollegen an den Ohren gezogen

Neben der Möglichkeit der ad-hoc-Metaphern gibt es aber für ziehen und seine Aktanten zahlreiche etablierte, lexikalisierte Verwendungsweisen, deren metaphorischer Charakter noch erkennbar ist, weil die dazugehörigen nicht-metaphorischen Verwendungsweisen gebräuchlich sind:

(65) Die Deutschen ziehen die Amerikaner bei den Verhandlungen uber den Tisch

(66) BMW zieht bei Rover die Notbremse

(67) Wollte Kohl nicht auch auf seine Weise einmal einen Schlussstrich ziehen?

(68) Er hat wieder einmal alle Register gezogen

(69) Suhartos Sippe zieht die Fáden

Daneben gibt es auch verfestigte idiomatische Wendungen, deren metaphorische Deutung den Sprechern im Allgemeinen nicht mehr zugänglich ist:

(70) Er zieht gewaltig vom Leder

(71) Zieh Leine!

Schließlich gibt es auch eine ganze Anzahl fachsprachlicher Verwendungsweisen, die wohl ebenfalls als isolierte Verwendungsweisen gelten müssen:

(72) Dann wird der Wein auf Flaschen gezogen

(73) Rustungslieferanten konnten auf die „Metallurgische Forschungsgesellschaft" (Mefo) Wechsel ziehen

Zusammen mit den zu Beginn erwähnten festen Verbindungen wie z. B. den Funktionsverbgefugen vom Typ in Erwägung ziehen findet sich also eine ganze Anzahl von Verwendungsweisen, die sozusagen außerhalb des Familienverbandes existieren und bei denen deshalb der Versuch nicht aussichtsreich erscheint, synchronisch Zusammenhänge mit anderen Verwendungsweisen zu rekonstruieren.

\section{Zur Verwandtschaft der Verwendungsweisen: ein Familienbild}

Nachdem ich nun einen Ausschnitt aus dem Spektrum der Verwendungsweisen von ziehen beschrieben habe, will ich die wichtigsten Beschreibungsergebnisse in einem Verwandtschaftsgraphen übersichtlicher darstellen. Zur einfacheren Kennzeichnung der Verwendungsweisen verwende ich Kürzel wie Verformung, die jeweils Hauptaspekte der betreffenden Beschreibung angeben. 


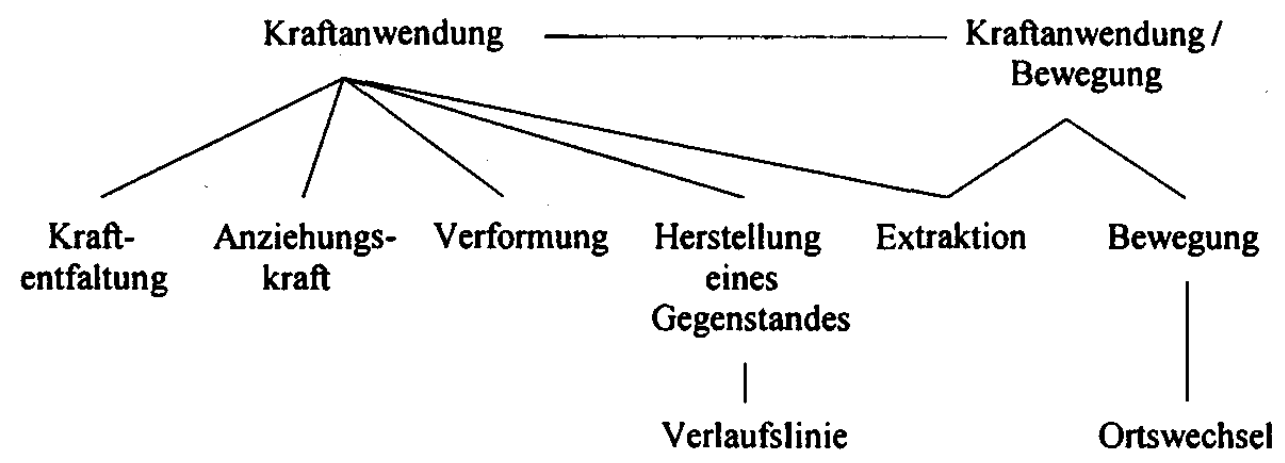

In diesem Graphen sind die feineren Verzweigungen nicht berücksichtigt, die in den vorigen Abschnitten eingeführt wurden. Wichtiger noch: Es sind mit der Ausnahme von Extraktion keine Mehrfachverbindungen dargestellt, so dass auf dieser Ebene der Beschreibung das System der Verwendungsweisen sich als Baum darstellt und nicht, wie bei feinkörnigerer Beschreibung, als Netz.

\section{Zusammenfassung: Semantische Verfahren, Wissensbestănde, Ökonomie}

Extreme Polysemie funktioniert, das zeigt der Fall ziehen, deshalb, weil die Sprecher uber semantische Verfahren und Wissensbestände verfugen, die es ihnen erlauben, Zusammenhänge zwischen Verwendungsweisen zu sehen und aktuelle Verwendungen auf der Folie unterschiedlicher etablierter Verwendungsweisen zu deuten. Allgemein verfügbare Verfahren zu diesem Zweck sind die Orientierung an Kommunikationsprinzipien wie dem der Relevanz und die Anwendung Gricescher Ableitungsverfahren. Speziell für den Gebrauch von ziehen haben sich die folgenden semantischen Techniken als grundlegend herausgestellt: ${ }^{9}$

- die Fokussierung von Ereignisaspekten,

- die Verlagerung des Fokus,

- die Einfuhrung neuer Aspekte,

- das Ausblenden von Aspekten,

- metaphorische Verwendung von Ausdrucken.

Beim Verfahren, einen Aspekt des Ereignistyps aus dem Fokus zu nehmen, ließen sich zwei Stufen unterscheiden: das implizite Voraussetzen eines Aspekts und das vollige Ausblenden des Aspekts. Die semantische Technik der Verlagerung des Fokus wird teilweise grammatisch realisiert durch elliptischen Gebrauch, durch die Einführung zusätzlicher Aktanten und durch Agensverschiebung. Es ist daher nicht verwunderlich, dass die Valenzeigenschaften des Verbs in meiner Beschreibung eine wichtige Rolle spielen. Als grundlegende Wissensbestände werden verwendet - ich wiederhole aus Abschnitt 2:

9 Die Fokussierung, Verlagerung, Einfuhrung und Ausblendung von Aspekten eines Ereignistyps konnte man als ein metonymisches Verfahren bezeichnen. Ich glaube aber, dass mit dieser Bezeichnung nicht viel gewonnen ist. 
(i) stereotypes Wissen uber eine Anzahl von grundlegenden Ereignistypen, die mit ziehen charakterisiert werden können, und deren Hauptaspekte,

(ii) stereotypes Wissen uber bestimmte Arten von Gegenständen (bewegliche Gegenstănde, elastische Gegenstănde, flüchtige Gegenstănde etc.).

Wenn diese Verfahren und Wissensbestände den Sprechern verfügbar sind, dann ist Polysemie, auch extreme Polysemie, nicht nur unproblematisch, sondern sogar funktional und okonomisch. Diese Auffassung steht im Einklang mit Bréals Einsicht, dass für die Sprachbenutzer eine zusătzliche Verwendungsweise so viel leistet wie ein zusătzliches Wort. ${ }^{10}$

10 Bréal, Michel: Essai de Sémantique. Science des Significations. 7. Auflage. Paris 1924, S. 146. 


\section{Sprachspiel und Bedeutung}

Festschrift für Franz Hundsnurscher zum 65. Geburtstag

Herausgegeben von

Susanne Beckmann, Peter-Paul König und Georg Wolf

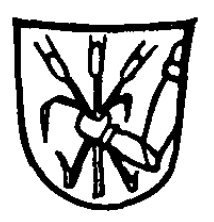

Max Niemeyer Verlag

Tübingen 2000 